\title{
Longitudinal assessment and stability of long non-coding RNA gene expression profiles measured in human peripheral whole blood collected into PAXgene blood RNA tubes
}

\author{
Lukasz S. Wylezinski ${ }^{1,2,3 \dagger}$, Guzel I. Shaginurova ${ }^{1 \dagger}$ and Charles F. Spurlock $\mathrm{III}^{1,2,3^{*}}$
}

\begin{abstract}
Objective: Long non-coding RNAs (IncRNAs) are emerging as novel biomarkers for a variety of chronic conditions including autoimmune disease. PAXgene Blood RNA tubes are routinely used in clinical research and molecular diagnostic development to capture RNA profiles in peripheral whole blood. While the stability of mRNA expression profiles captured using PAXgene tubes has been documented previously, no previous work has determined the stability of IncRNA expression profiles observed in PAXgene tubes stored at $-80^{\circ} \mathrm{C}$. Here we sought to determine the effects on IncRNA expression profiles following $-80^{\circ} \mathrm{C}$ storage of total RNA templates, CDNA synthesized using fresh or frozen total RNA template, and the impact of freeze-thaw cycles on both total RNA and cDNA obtained from PAXgene tubes.
\end{abstract}

Results: We find that storage of whole blood in PAXgene tubes, total RNA and CDNA for up to 1 year at $-80^{\circ} \mathrm{C}$ or up to ten total RNA or cDNA freeze-thaw cycles do not significantly alter IncRNA expression profiles compared to baseline. As monthly expression profiles were determined, some month to month IncRNA expression variability was observed. However, all monthly observations fell within the 95\% confidence interval calculated at baseline.

Keywords: Long non-coding RNA, Multiple sclerosis, Messenger RNA, PAXgene blood RNA tube, Quantitative real time PCR, Storage

\section{Introduction}

Long non-coding RNAs (lncRNAs) play pivotal roles in gene regulation, protein synthesis, sex chromosome compensation and telomere maintenance [1-5]. Work over the past decade has also implicated specific lncRNAs in a variety of pathological processes and human diseases including cancer, autoimmune disease and neurodegenerative disorders like Parkinson's and Alzheimer's [6-13]. Apart from mechanistic studies ascribing

*Correspondence: chase@iquity.com

${ }^{\dagger}$ Lukasz S. Wylezinski and Guzel I. Shaginurova contributed equally to this work.

${ }^{1}$ IQuity, Inc, 111, 10th Avenue South, Suite 100, Nashville, TN 37203, USA Full list of author information is available at the end of the article biological outcomes to over- or under-expression of specific lncRNAs, lncRNAs that exhibit expression differences are often proposed as candidate biomarkers that could be measured in novel RNA-based assays to aid in the diagnosis of disease or to monitor disease progression [14-17]. Our own cross-sectional studies in human disease have included extensive RNA sequencing and quantitative real time PCR (qRT-PCR) studies to measure a variety of RNA species including protein-coding and non-coding genes, with a particular focus on lncRNAs. We identified a series of mRNAs, annotated and novel lncRNA genes that are differentially expressed among relapsing-remitting multiple sclerosis patients, healthy control subjects, and disease controls [16,

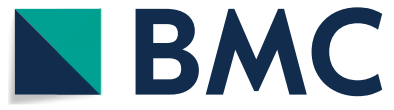

(c) The Author(s) 2020. This article is licensed under a Creative Commons Attribution 4.0 International License, which permits use, sharing, adaptation, distribution and reproduction in any medium or format, as long as you give appropriate credit to the original author(s) and the source, provide a link to the Creative Commons licence, and indicate if changes were made. The images or other third party material in this article are included in the article's Creative Commons licence, unless indicated otherwise in a credit line to the material. If material is not included in the article's Creative Commons licence and your intended use is not permitted by statutory regulation or exceeds the permitted use, you will need to obtain permission directly from the copyright holder. To view a copy of this licence, visit http://creativeco mmons.org/licenses/by/4.0/. The Creative Commons Public Domain Dedication waiver (http://creativecommons.org/publicdomain/ zero/1.0/) applies to the data made available in this article, unless otherwise stated in a credit line to the data. 
18-20]. These expression differences were measured in peripheral whole blood samples collected into PAXgene Blood RNA tubes. Previous studies have demonstrated the relative stability of messenger RNA (mRNA) expression after PAXgene tube storage at $-70{ }^{\circ} \mathrm{C}$ [21] for up to 11 years [22]. To our knowledge, no previous research has determined whether extended storage of whole blood collected into PAXgene tubes could alter lncRNA expression. Examining whether differences in lncRNA expression measurements are produced from cDNA synthesized using freshly isolated total RNA template or total RNA template stored at $-80^{\circ} \mathrm{C}$ for periods of up to 1 year is also incompletely understood. Additionally, no previous work has documented the impact of multiple freeze-thaw cycles of total RNA template or synthesized cDNA on lncRNA qRT-PCR studies. Given the potential utility of lncRNAs in peripheral whole blood to serve as biomarkers for disease, we conducted a 1 year stability study of lncRNA expression profiles using peripheral whole blood collected and stored in PAXgene tubes.

\section{Main text \\ Methods \\ Blood collection, RNA isolation, CDNA synthesis, and nucleic acid assessment}

RNA was isolated from peripheral whole blood collected into PAXgene Blood RNA tubes (PreAnalytiX) according to the manufacturer's supplied protocol using an automated QIAcube system (Qiagen). RNA samples were further purified, concentrated, and eluted in RNase-free water using the RNEasy MinElute Cleanup Kit (Cat. No. 74204, Qiagen). cDNA synthesis was performed using the Superscript III First-Strand Synthesis System with oligo-dT as primer (Cat. No. 18080051, Invitrogen). cDNA samples were purified and eluted in elution buffer (10 mM TrisCl, $\mathrm{pH}=8.5)$ using the QiaQuick PCR Purification Kit (Cat. No. 28106, Qiagen) prior to qRT-PCR measurements. Total RNA and cDNA were assessed for quantity and purity using a Nanodrop 1000 spectrophotometer (ThermoFisher) prior to storage at $-80{ }^{\circ} \mathrm{C}$ in $1.5 \mathrm{~mL}$ Eppendorf tubes. RNA integrity number (RIN) values were obtained using the RNA 6000 Nano Total RNA Kit and a Bioanalyzer 2100 instrument (Cat. No. 5067-1511, Agilent Technologies, Inc.). All above-mentioned steps were performed according to the manufacturer's protocols. Freeze-thaw studies were performed by thawing RNA samples and incubating the sample for $10 \mathrm{~min}$ at room temperature $\left(21^{\circ} \mathrm{C}\right)$ and re-freezing the sample at $-80^{\circ} \mathrm{C}$ for $30 \mathrm{~min}$.

\section{qRT-PCR measurements}

Custom DNA oligos were synthesized (Integrated DNA Technologies, Inc.) that target specific lncRNA and
mRNA genomic loci (Additional file 1: Table S1). Quality control steps were performed prior to qRT-PCR studies. Single PCR products were confirmed by agarose gel electrophoresis and amplified PCR products were verified by Sanger Sequencing (Genewiz) (data not shown). qRT-PCR measurements were performed using a QuantStudio 12 K Flex PCR system (Life Technologies). cDNA samples were loaded into 384 -well plates in a $10 \mu \mathrm{L}$ final volume with PowerUp SYBR Green Master Mix (Applied Biosystems) and a final primer concentration of $0.5 \mu \mathrm{M}$. 384-well plates were pre-loaded with primers using automated liquid handler VIAFLO96/384 (Integra Biosciences Corp.). Expression values were normalized to GAPDH using the $\triangle \triangle \mathrm{Ct}$ method as previously described $[23,24]$.

\section{Statistical analysis}

Statistical analysis was performed using GraphPad Prism 8.3.1 and denoted within each figure legend.

\section{Results \\ Evaluation of IncRNA expression following storage of PAXgene tubes, RNA and CDNA for up to one year}

To examine the effects of various storage conditions on lncRNA expression, we initiated our study utilizing a panel of annotated lncRNAs from previous RNA sequencing studies that were conducted across multiple human autoimmune diseases (Additional file 1: Table S1 and Additional file 2: Fig.S1). These studies identified annotated lncRNAs that were differentially expressed among multiple sclerosis and healthy control subjects and were further validated by qRT-PCR measurements [18]. Six of these annotated IncRNAs were selected to assess the stability of RNA transcripts across various storage conditions: PAXgene tube, purified total RNA, and synthesized cDNA. A schematic of experimental steps is presented in Additional file 3: Fig.S2. Peripheral whole blood from five healthy donors was collected into twelve PAXgene tubes per individual. Samples were pooled to create a homogenous mixture and then aliquoted equally into sixty tubes. Total RNA was immediately isolated from five PAXgene tubes, cDNA was synthesized and expression levels of the six annotated lncRNAs measured to establish a baseline expression profile. The additional tubes were frozen at $-80^{\circ} \mathrm{C}$ for use at future timepoints. The remaining eleven experimental groups of five PAXgene tubes were stored and isolated each month over the course of 1 year. Expression of annotated lncRNAs isolated at baseline were compared to expression of lncRNAs from PAXgene tubes where RNA was isolated and cDNA was synthesized 1 year later. Whether total RNA was isolated from PAXgene tubes immediately or whole blood lysates were stored with total RNA purified 1 year 
later, no statistically significant differences in lncRNA expression were found (Fig. 1a). Stability of purified total RNA (Fig. 1b) and cDNA (Fig. 1c) was then compared at baseline and after storage for 1 year. Similar to what was observed in RNA isolated immediately or after 1 year from each PAXgene tube, no significant difference was observed across the six annotated lncRNAs we measured following storage of total RNA or cDNA.

During the course of the study, consecutive, monthly assessments were also performed whereby five PAXgene tubes were pulled from storage and expression levels of each lncRNA was analyzed for a total of twelve series of qRT-PCR measurements (Additional file 4: Fig. S3). Across each monthly experiment, the $95 \%$ CI was calculated and compared to baseline lncRNA expression values after normalization to GAPDH. No significant differences were observed for RP11-97C16.1 or LINC00847. However, significant differences in the 95\% CI were observed at month 10 for RP11-125,214.2, AC012314.8, and RP11-335112.2. Additionally, a significant difference in the $95 \% \mathrm{CI}$ was observed in months 2, 4, 6, and 7 for MCCC1-AS1. On a monthly basis we saw a stochastic pattern of IncRNA expression oscillating around the baseline level established. IncRNAs with lower expression levels, in particular MCCC1-AS1, with a mean Ct value of 29.73, exhibited multiple months of statistically significant differences in expression. When baseline values were compared to each monthly observation, none of the lncRNAs fell outside of the $95 \%$ baseline CI established at the start of the study. Furthermore, in five of the six lncRNAs, all monthly $95 \%$ CI overlapped. In MCCC1-AS1, however, the $95 \% \mathrm{CI}$ at months $2,4,6$, and 7 did not overlap when these individual months were compared indicating a pattern of greater variability in this particular gene target. This is consistent with other studies that have noted greater experimental variation among genes with higher $\mathrm{Ct}$ values [25]. Furthermore, it has been shown that lncRNAs display cell type specificity as well as a wide range of intrinsic stability [26, 27]. Inherently, more stable lncRNAs will be better equipped to endure storage conditions. These differences become more pronounced when measuring lncRNAs expressed at low levels. We believe this phenomenon is observed in the variability of MCCC1-AS1 measurements as compared to $A C 012314.8$.

Quality metrics for all RNA samples processed during the study were also assessed (Additional file 5: Table S2). Each PAXgene tube containing pooled blood from five donors yielded, on average, between 2.72 and $7.98 \mu \mathrm{g}$ of purified total RNA with no discernible correlation between length of storage at $-80{ }^{\circ} \mathrm{C}$ and total amount of RNA isolated (Additional file 6: Fig.S4). During the course of our study, the coefficient of variation calculated for RNA yield ranged from $4.41 \%$ to $19.37 \%$ (Additional file 6: Fig.S4d). This falls within the range previously reported by the manufacturer and cleared by the Federal Drug Administration in its approval of PAXgene tubes as medical devices [28].

\section{Evaluation of RNA integrity number, IncRNA and mRNA expression following freeze-thaw cycles}

We next investigated the effects of long-term storage on RNA integrity and the impact of multiple freeze-thaw cycles on total RNA by analyzing RNA integrity number (RIN) values as depicted in Additional file 7: Fig.S5. These values are calculated comparing $18 \mathrm{~S}$ and $28 \mathrm{~S}$ rRNA ratios a

Baseline PAXgene tube PAXgene tube stored for 1 year

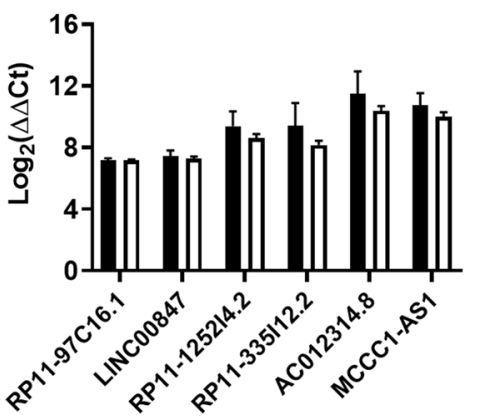

b

$$
\square \text { RNA stored for } 1 \text { year }
$$

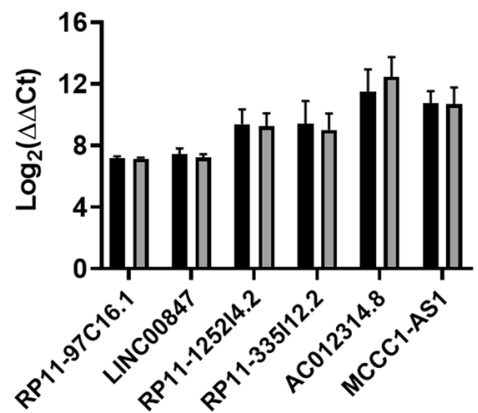

C

Baseline CDNA $\boldsymbol{\nabla}$ cDNA stored for 1 year

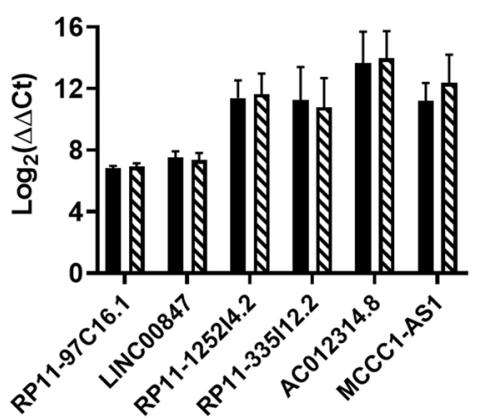

Fig.1 Annotated IncRNA expression values do not differ significantly after storage for 1 year at $-80^{\circ} \mathrm{C}$. IncRNA expression levels were measured using a total RNA immediately isolated from PAXgene tubes at baseline or RNA isolated after PAXgene tubes were stored at $-80^{\circ} \mathrm{C}$ for one year, $\mathbf{b}$ total RNA isolated at baseline compared to the same RNA stored at $-80^{\circ} \mathrm{C}$ for one year, or c CDNA immediately prepared using baseline total RNA or the same cDNA sample stored for one year at $-80^{\circ} \mathrm{C}$. Student's t-test with Welch's correction was used to determine statistical significance. Bars represent mean $\log _{2}(\Delta \Delta \mathrm{Ct})$ with SD for $\mathrm{n}=5$ PAXgene tubes with pooled blood, RNA samples or cDNA samples in each experimental group 
where values greater than or equal to 7 are generally considered acceptable [29]. RNA samples isolated at baseline and stored for 1 year at $-80{ }^{\circ} \mathrm{C}$ exhibited no significant difference in overall RNA integrity determined by RIN (Fig. 2). Similarly, RNA samples subjected to five or ten freeze-thaw cycles did not show any significant degradation when analyzed by RIN values. Digitally captured electropherograms of total RNA samples used for RIN analysis are shown in Additional file 8: Fig.S6.

To further assess the quality of each RNA template, cDNA was synthesized from each of the RNA samples at baseline, after storage for 1 year or after a series of five or ten freeze-thaw cycles. cDNA synthesized from each of these RNA templates did not exhibit significant differences in lncRNA expression values (Fig. 3a). As an additional comparison, canonical protein-coding genes were also measured to test whether $-80{ }^{\circ} \mathrm{C}$ storage or up to 10 freeze-thaw cycles would significantly alter expression of these mRNA targets as well (Fig. 3b). These mRNAs were selected from the same study comparing healthy control subjects and patients with relapsing-remitting multiple sclerosis $[16,18]$. Just as we observed in lncRNAs, no significant differences in mRNA expression were found. Mean cycle threshold $(\mathrm{Ct})$ values $\pm \mathrm{SD}$ were compared across each experimental group. Amplification of all gene targets resulted in gene targets with $\mathrm{Ct}$ values that were less than 29. No significant difference in $\mathrm{Ct}$ value was observed across each of the experimental comparisons (Additional file 9: Fig.S7). Finally, similar results were also observed when cDNA samples were exposed to five or ten freeze-thaw cycles (Additional file 10: Fig.S8).

\section{Discussion}

Since the beginning of this century, the number of documented RNA classes, and variety of noncoding transcripts has grown significantly. In the mid-2000s, the FANTOM3 project identified $\sim 35,000$ non-coding transcripts from $\sim 10,000$ distinct loci [30]. Recently a comprehensive analysis of lncRNAs from existing databases, published literature and novel RNA assemblies revealed that there are 270,044 lncRNA transcripts in the human genome [31]. PAXgene tubes are widely used in biobanking initiatives, clinical research studies, and molecular diagnostics to stabilize RNA species. Since PAXgene tubes are used with increasing frequency to correlate disease outcomes with RNA transcript levels, careful consideration should be given to downstream diagnostic applications to ensure reproducibility of RNA measurements over time.

We find that PAXgene tubes maintain the stability of lncRNA expression profiles when stored at $-80^{\circ} \mathrm{C}$ for up to 1 year. We also find that storing total RNA or cDNA synthesized from fresh total RNA template or total RNA stored at $-80{ }^{\circ} \mathrm{C}$ for 1 year does not result in significant changes in lncRNA expression. While some month to month variation between datapoints is observed, we believe that these changes are inherent in the technical processing of samples and can

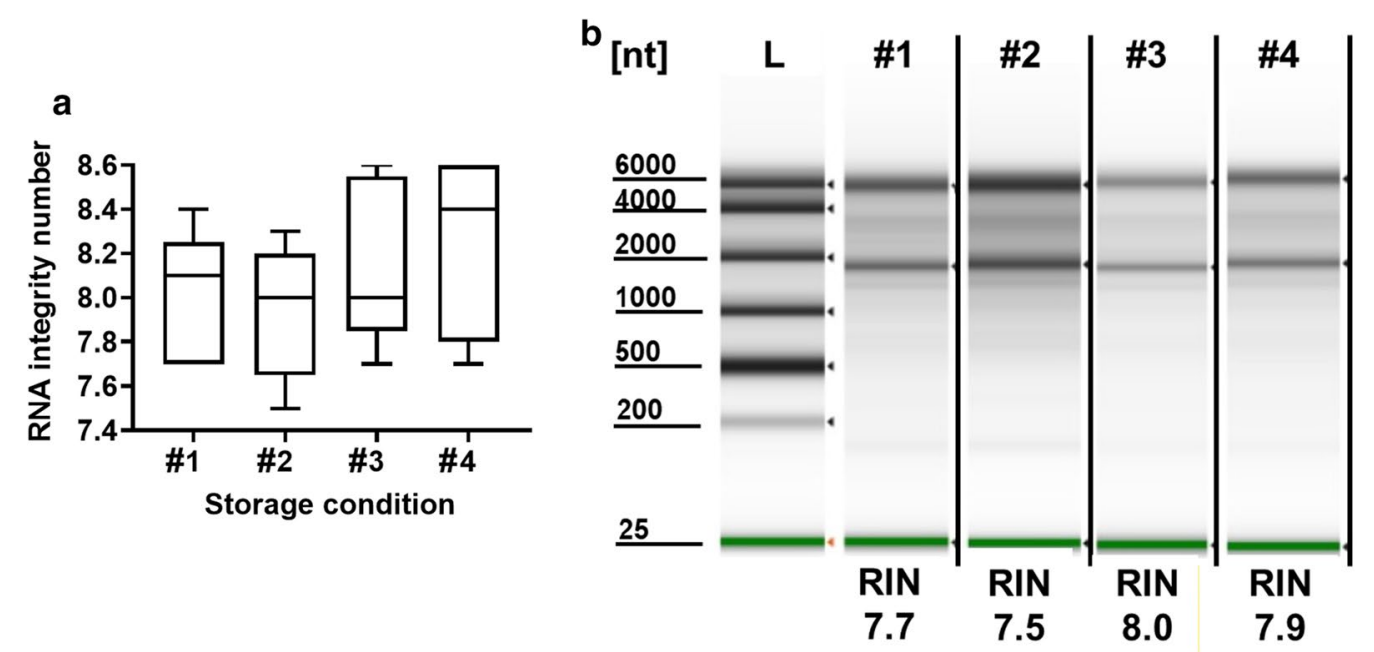

Fig. 2 Long term storage and freeze-thaw cycles do not significantly impact RNA isolated from PAXgene tubes. The following conditions with corresponding numbers are represented in this figure: \#1—baseline RNA that was immediately isolated; \#2—isolated RNA stored at - $80^{\circ} \mathrm{C}$ for one year; \#3-isolated RNA exposed to five freeze-thaw cycles; \#4-isolated RNA exposed to ten freeze-thaw cycles. a mean RIN values with SD for $n=5$ healthy controls for each condition. $\mathbf{b}$ electropherogram and RIN values of a representative subject's total RNA samples for each condition, L RNA ladder, nt nucleotide length. RIN analysis and electropherogram visualization were performed on the same gel and on the same day for all treatment conditions 
Baseline RNA

RNA stored for 1 year

a
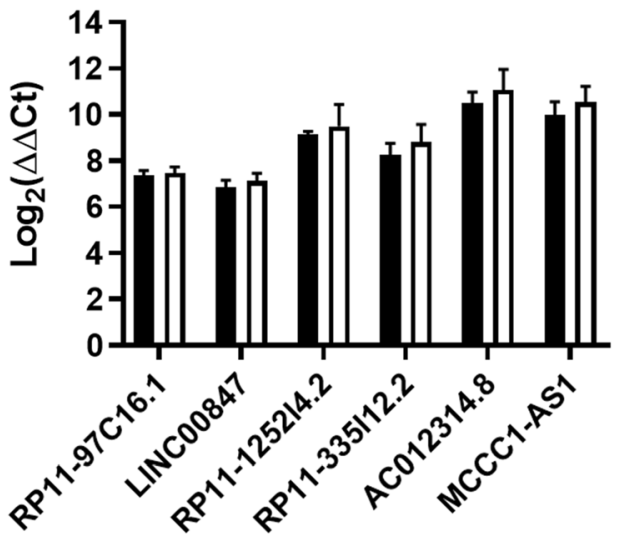

b

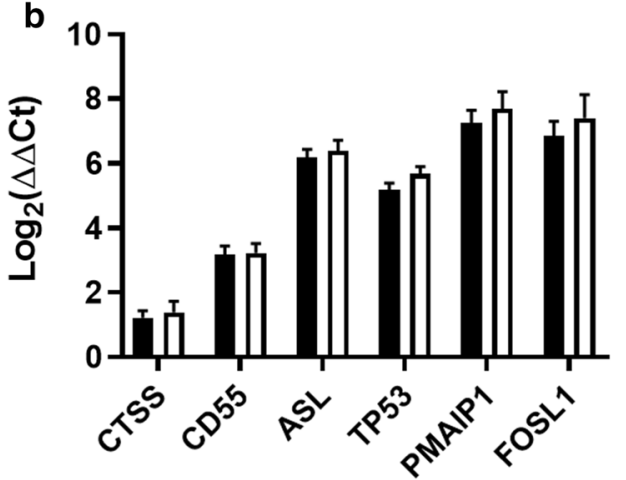

Baseline RNA

$\mathbf{\Delta}$ RNA after 5 freeze-thaw cycles

RNA after 10 freeze-thaw cycles
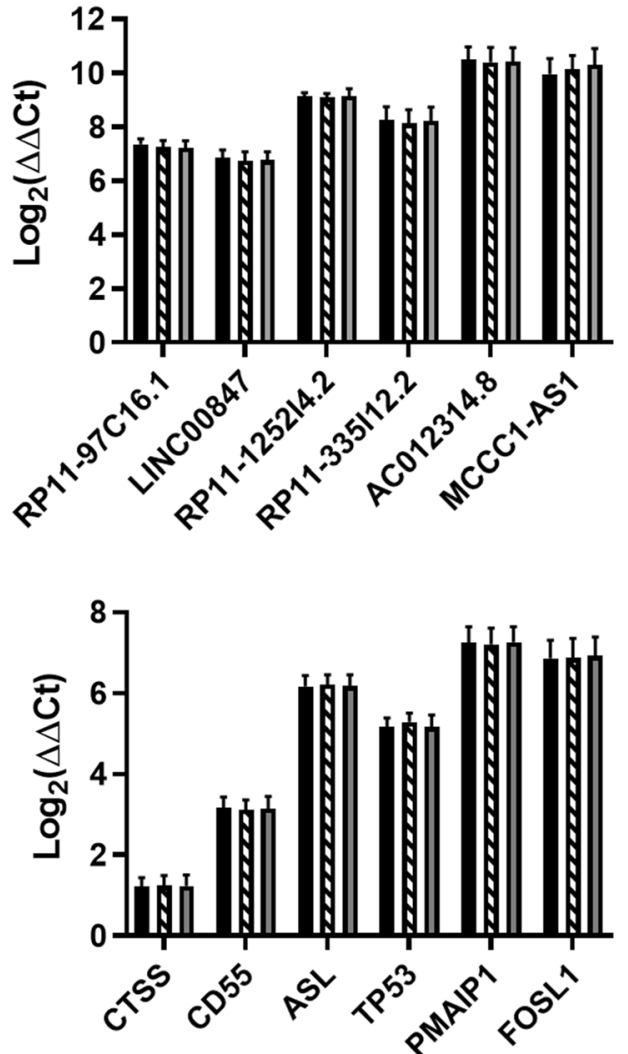

Fig. 3 Long term storage and freeze-thaw cycles do not alter IncRNA or mRNA expression. qRT-PCR gene expression data were produced for a six IncRNAs and $\mathbf{b}$ six mRNAs. Baseline RNA immediately isolated from PAXgene Blood RNA tubes was compared to three treatment conditions including RNA storage at $-80^{\circ} \mathrm{C}$ for one year, total RNA from PAXgene tubes exposed to five freeze-thaw cycles, and total RNA that was exposed to ten freeze-thaw cycles. Paired sample t-test was used to determine statistical significance. Bars represent mean $\log _{2}(\Delta \triangle C t)$ with $S D$ for $n=5$ individual healthy subject's baseline total RNA samples and after one of the three treatment conditions

disproportionately affect qRT-PCR targets of lower abundance. Additionally, while not directly examined in our work, previous studies have shown that expression measurements using shorter target amplicon lengths (70-250 bp) are less susceptible to variations in RNA integrity than larger PCR amplification products [29]. With the exception of MCCC1-AS1 in our analysis, the monthly fluctuations observed among other lncRNAs resulted in overlapping 95\% confidence intervals. Future studies seeking to develop novel assays based on mRNA or IncRNA expression values should examine expression differences over time, particularly for transcripts with lower overall expression values, to ensure that any technical variation or intrinsic stability does not adversely impact the accuracy, reproducibility or interpretability of assay results.

\section{Limitations}

While the characteristics of each respective target should be assessed individually, one limitation of this study was the relatively small number of lncRNA and mRNA targets evaluated. Three laboratory technicians performed PAXgene RNA isolations, synthesized cDNA, and performed gene expression studies. This was intentional to simulate multiple operators that could potentially process PAXgene tubes in a clinical setting. PCR primers used in this study were printed onto 384-well plates robotically. Additional liquid handling steps could be implemented to further minimize operator to operator technical variation.

lncRNA and mRNA expression was normalized to $G A P D H$ throughout our investigation. While GAPDH is widely accepted as an appropriate housekeeping gene, depending upon the use case, incorporation of 
other housekeeping genes could further reduce operator to operator or monthly expression variability. While single RIN assessments were performed for each of the experimental conditions in Fig. 2, these measurements were not performed for each sample across all experimental conditions in the study. Thus, we are unable to determine whether slight variations in RIN values could account for expression variability.

\section{Supplementary information}

Supplementary information accompanies this paper at https://doi. org/10.1186/s13104-020-05360-3. Additional file 1: Table S1. Genomic locations for qRT-PCR targets used
in the study.

Additional file 2: Figure S1. RNA sequencing data for annotated IncRNAs differentially expressed among healthy controls and MS patients.

Additional file 3: Figure S2. Schematic representation of the IncRNA stability study.

Additional file 4: Figure S3. Monthly IncRNA expression analysis over the course of 1 year.

Additional file 5: Table S2. Summary of quality metrics across all total RNA samples isolated from PAXgene Blood RNA tubes.

Additional file 6: Figure S4. Summary of total RNA yield from PAXgene Blood RNA tubes processed during the 1 year study.

Additional file 7: Figure S5. Schematic representation of freeze-thaw experiments.

Additional file 8: Figure S6. Digitally captured full size electropherogram of total RNA samples processed for RNA Integrity Number analysis.

Additional file 9: Figure S7. Summary of IncRNA and mRNA expression values and representative amplification plots for mRNAs and IncRNAs.

Additional file 10: Figure S8. Long term cDNA storage and freeze-thaw cycles do not alter IncRNA or mRNA expression.

\section{Abbreviations}

Cl: Confidence interval; CV: Coefficient of variation; FC: Fold change; FPKM: Fragments per kilobase of transcript per million mapped reads; GAPDH: Glyceraldehyde 3-phosphate dehydrogenase; IncRNA: Long non-coding RNA; mRNA: Messenger RNA; MS: Multiple sclerosis; qRT-PCR: Quantitative real-time polymerase chain reaction; RIN: RNA integrity number; SD: Standard deviation; SEM: Standard error of the mean.

\section{Acknowledgements}

The authors would like to thank the volunteers who participated in this study and provided blood specimens. We would also like to thank John Handshoe and John Tossberg for assistance processing blood samples.

\section{Authors' contributions}

Conception and study design: LSW, CFS. Data collection: GIS. Data analysis: LSW, GIS, CFS. All authors read and approved the final manuscript.

\section{Funding}

This work was supported by IQuity, Inc., grants from the National Institutes of Health (Al124766, Al129147, and Al145505), and the Launch Tennessee SBIR/ STTR Matching Fund.

\section{Availability of data and materials}

The datasets used and/or analyzed during the current study are available from the corresponding author upon reasonable request. Previous RNA-sequencing data used to define PCR targets are publicly available [18].

\section{Ethics approval and consent to participate}

Protocols for obtaining volunteer blood samples were reviewed and approved by the Western Institutional Review Board (WIRB20171157). Study participants provided written informed consent.

\section{Consent for publication}

Not applicable.

\section{Competing interests}

IQuity, Inc. is a company focused on developing RNA-based tools to aid in the diagnosis of chronic diseases, including autoimmune disease.

\section{Author details}

${ }^{1}$ IQuity, Inc, 111, 10th Avenue South, Suite 100, Nashville, TN 37203, USA.

2 Decode Health, Inc, 209 10th Avenue South, Suite 404, Nashville, TN, USA.

${ }^{3}$ Department of Medicine, Vanderbilt University School of Medicine, 1161 21st Avenue South, Medical Center North T3113, Nashville, TN, USA.

Received: 17 March 2020 Accepted: 26 October 2020

Published online: 12 November 2020

\section{References}

1. Salta E, De Strooper B. Noncoding RNAs in neurodegeneration. Nat Rev Neurosci. 2017;18(10):627-40.

2. Chen YG, Satpathy AT, Chang HY. Gene regulation in the immune system by long noncoding RNAs. Nat Immunol. 2017;18(9):962-72.

3. Mumbach MR, et al. HiChIRP reveals RNA-associated chromosome conformation. Nat Methods. 2019;16(6):489-92.

4. Kung JT, Colognori D, Lee JT. Long noncoding RNAs: past, present, and future. Genetics. 2013;193(3):651-69.

5. Dey BK, Mueller AC, Dutta A. Long non-coding RNAs as emerging regulators of differentiation, development, and disease. Transcription. 2014;5(4):e944014.

6. DiStefano JK. The Emerging Role of Long Noncoding RNAs in Human Disease. Methods Mol Biol. 2018;1706:91-110.

7. Simchovitz $A$, et al. A IncRNA survey finds increases in neuroprotective LINC-PINT in Parkinson's disease substantia nigra. Aging Cell. 2020;19:e13115.

8. Wang DQ, et al. Long non-coding RNAs, novel culprits, or bodyguards in neurodegenerative diseases. Mol Ther Nucleic Acids. 2018;10:269-76.

9. Li J, et al. Comprehensive analysis of therapy-related messenger RNAs and long noncoding RNAs as novel biomarkers for advanced colorectal cancer. Front Genet. 2019;10:803.

10. Sathishkumar $C$, et al. Linking a role of IncRNAs (long non-coding RNAs) with insulin resistance, accelerated senescence, and inflammation in patients with type 2 diabetes. Hum Genomics. 2018;12(1):41.

11. Tang $Y$, et al. The role of long non-coding RNAs in rheumatic diseases. Nat Rev Rheumatol. 2017;13(11):657-69.

12. Riva P, Ratti A, Venturin M. The long non-coding RNAs in neurodegenerative diseases: novel mechanisms of pathogenesis. Curr Alzheimer Res. 2016;13(11):1219-31.

13. Hur K, Kim SH, Kim JM. Potential implications of long noncoding RNAs in autoimmune diseases. Immune Netw. 2019;19(1):e4.

14. Van Roosbroeck K, Pollet J, Calin GA. miRNAs and long noncoding RNAs as biomarkers in human diseases. Expert Rev Mol Diagn. 2013;13(2):183-204

15. Spurlock CF, et al. Methotrexate inhibits NF-kB activity via long intergenic (noncoding) RNA-p21 induction. Arthritis Rheumatol. 2014;66(11):2947-57

16. Spurlock, C.F. A molecular-based approach using long, non-coding RNA and enhancer-associated IncRNA gene expression signatures to classify multiple sclerosis using peripheral whole blood. In: ECTRIMS. 2017. Paris: ECTRIMS Online. https://onlinelibrary.ectrims-congress.eu/ectrims/2017/ ACTRIMS-ECTRIMS2017/199954/charles.f.spurlock.iii.a.molecular-based .approach.using.long.non-coding.rna.html.

17. Franco-Acevedo A, Melo Z, Echavarria R. Diagnostic, prognostic, and therapeutic value of non-coding RNA expression profiles in renal transplantation. Diagnostics (Basel). 2020;10(2):60. 
18. Aune TM, et al. Expression of long non-coding RNAs in autoimmunity and linkage to enhancer function and autoimmune disease risk genetic variants. J Autoimmun. 2017:81:99-109.

19. Tossberg JT, et al. Gene-expression signatures: biomarkers toward diagnosing multiple sclerosis. Genes Immun. 2012;13(2):146-54.

20. Tossberg JT, et al. Using biomarkers to predict progression from clinically isolated syndrome to multiple sclerosis. J Clin Bioinforma. 2013;3(1):18.

21. Häntzsch M, et al. Comparison of whole blood RNA preservation tubes and novel generation RNA extraction kits for analysis of mRNA and MiRNA profiles. PLoS ONE. 2014;9(12):e113298.

22. PAXgene Blood RNA System. Technical Note, 2018. 2020. https://www preanalytix.com/storage/download/_ProductResources_/TechnicalN otes/PROM-7266-002_BD-7969_TN_Blood_RNA_System_RNA_stabi lity_over_11_years_storage_1118_WW_WEB.pdf. Accessed 2020 Feb 26.

23. Green MR, Sambrook J. Analysis and normalization of real-time polymerase chain reaction (PCR) experimental data. Cold Spring Harb Protoc. 2018. https://doi.org/10.1101/pdb.top095000.

24. Zhang $F$, et al. Expression of IL-33 and its epigenetic regulation in multiple sclerosis. Ann Clin Transl Neurol. 2014;1(5):307-18.

25. Kuang J, et al. An overview of technical considerations when using quantitative real-time PCR analysis of gene expression in human exercise research. PLoS ONE. 2018;13(5):e0196438.
26. Clark MB, et al. Genome-wide analysis of long noncoding RNA stability. Genome Res. 2012;22(5):885-98.

27. Tani H, et al. Genome-wide determination of RNA stability reveals hundreds of short-lived noncoding transcripts in mammals. Genome Res. 2012;22(5):947-56.

28. PreAnalytix. PAXgene blood RNA Kit handbook. Hombrechtikon: PreAnaIytix; 2009. p. 55.

29. Fleige S, Pfaffl MW. RNA integrity and the effect on the real-time qRT-PCR performance. Mol Aspects Med. 2006;27(2-3):126-39.

30. Carninci $P$, et al. The transcriptional landscape of the mammalian genome. Science. 2005;309(5740):1559-63.

31. Ma L, et al. LncBook: a curated knowledgebase of human long noncoding RNAs. Nucleic Acids Res. 2019;47(5):2699.

\section{Publisher's Note}

Springer Nature remains neutral with regard to jurisdictional claims in published maps and institutional affiliations.
Ready to submit your research? Choose BMC and benefit from:

- fast, convenient online submission

- thorough peer review by experienced researchers in your field

- rapid publication on acceptance

- support for research data, including large and complex data types

- gold Open Access which fosters wider collaboration and increased citations

- maximum visibility for your research: over $100 \mathrm{M}$ website views per year

At BMC, research is always in progress.

Learn more biomedcentral.com/submissions 